\title{
Breast Columnar Cell Mucinous Carcinoma
}

National Cancer Institute

\section{Source}

National Cancer Institute. Breast Columnar Cell Mucinous Carcinoma. NCI Thesaurus. Code 40355.

An invasive breast adenocarcinoma characterized by the presence of tall columnar neoplastic cells that contain intracytoplasmic mucin. Grossly, cystic changes are not identified. 